\title{
Annäherungen an Covid-19 und Ungleichheiten in Lateinamerika $^{1}$
}

\author{
Alba Carosio
}

\section{Merkmale der Ungleichheit in Lateinamerika}

Die Covid-19 Pandemie erreichte Lateinamerika etwa Mitte März 2020. Es folgten social distancing, Lockdown und wirtschaftliche Lähmung. Das Virus traf auf eine bereits von tiefen strukturellen Ungleichheiten geprägte Region. Nach Angaben der Wirtschaftskommission für Lateinamerika und die Karibik (CEPAL) verzeichnete die Region seit 2014 einen Anstieg der Armut. Im Jahr 2019 lag der Anteil der in Armut ${ }^{2}$ lebenden Bevölkerung bei 30,8 Prozent und der Anteil der in extremer Armut $^{3}$ lebenden Bevölkerung bei 11,5 Prozent. In ländlichen Gebieten erreichte die Armut einen Höchstwert von 45,2 Prozent. Auch die mittleren Einkommensschichten sind von verschiedenen Benachteiligungen, Verwundbarkeiten und Risiken in Schlüsselbereichen wie Bildung, Arbeit und soziale Sicherung betroffen.

Ein seit den 1990er Jahren maßlos angewandtes und im zweiten Jahrzehnt des 21. Jahrhunderts wieder aufgenommenes Modell der Haushaltsdisziplin hat zum Rückzug des Staates aus der Bereitstellung grundlegender Daseinsvorsorge geführt. Wie David Harvey (2005) in seiner Analyse der Akkumulation durch Enteignung dargelegt hat, wurden in der neo-

1 Dieser Text ist das Ergebnis einer Forschungsarbeit, die ich mit Unterstützung des Centro Maria Sibylla Merian de Estudios Latinoamericanos Avanzados (CALAS) im Rahmen des Laboratoriums zur Bekämpfung der Ungleichheiten in Lateinamerika: Perspektiven auf Reichtum und Macht („Laboratorio Confrontando las desigualdades en América Latina: Perspectivas sobre riqueza y poder“) durchgeführt habe.

2 „Armut" wird definiert als eine Situation, in der das Einkommen geringer ist als der Wert eines Warenkorbs mit Grundnahrungsmitteln und anderen Dienstleistungen.

3 Unter "extremer Armut" versteht man eine Situation, in der keine Mittel zur Deckung des Grundbedarfs an Nahrungsmitteln vorhanden sind. Mit anderen Worten: Menschen, die in Haushalten leben, deren Einkommen nicht ausreicht, um einen Grundnahrungsmittelkorb zu kaufen, selbst wenn sie alles für Lebensmittel ausgeben, gelten als „extrem arm“. 
liberalen Phase Spekulation, ökologischer Raubbau, Betrug und private Aneignung eines wachsenden Teils des gesellschaftlichen Reichtums zur neuen Grundlage der Akkumulation. Dies blieb nicht ohne Konsequenzen: Die Pandemie offenbart die Prekarität der Arbeit in unserer Region. Kennzeichnend für unsere Gesellschaften ist ein weit verbreiteter Mangel an sozialer Sicherheit. Dies ist das Resultat der Kopplung wohlfahrtsstaatlicher Leistungen an formelle Beschäftigung sowie von nicht-universellen Sozialprogrammen und -politiken mit unterschiedlichen Formen der Fokussierung und der Privatisierung von Renten, Gesundheit und Bildung (Burchardt/Groisman 2014). Die Wohlfahrtsstaatlichkeit ist stark segmentiert: Die nicht als arm eingestufte Bevölkerung muss Marktleistungen in den Bereichen Gesundheit, Rente und Bildung einkaufen, da die Absicherung gegen Risiken als individuelle Verantwortung der Mittelschicht verstanden wird. Stratifizierte Wohlfahrtsstaaten haben zur (a) Kommodifizierung für bestimmte soziale Gruppen, (b) gezielten und selektiven Dekommodifizierung für die verarmten sozialen Gruppen und (c) einer erneuten Gewöhnung an familiäre Absicherung geführt. Angesichts der historischen Versäumnisse der Staaten, der Bevölkerung Wohlstand zu bieten, sind die Familien die wichtigste Institution, auf die Lateinamerikaner*innen sowohl kulturell als auch materiell zählen.

Mehr als die Hälfte (53 Prozent) der Erwerbstätigen in der Region, arbeitet im informellen Sektor: Von den insgesamt 292 Millionen Erwerbstätigen in Lateinamerika und der Karibik sind 158 Millionen informell beschäftigt ${ }^{4}$ (ILO 2020). Dies bedeutet niedrige Löhne und fehlende soziale Absicherung, da soziale Sicherheit und wohlfahrtsstaatliche Leistungen an die formelle Beschäftigung gebunden sind. Acht von zehn Erwerbstätigen in den ärmsten Schichten arbeiten ohne jegliche soziale Absicherung. Die Lockdown-Maßnahmen hatten verheerende Auswirkungen auf diese Bevölkerungsgruppe, da sie kein Einkommen zum Überleben generieren konnten. Home Office ist aufgrund der Merkmale ihrer Arbeitsplätze, ihrer Ausbildung, der Ausstattung und der fehlenden oder prekären Internetverbindungen in der gesamten Region keine Option für die Ärmsten. Quarantäne und soziale Isolation bedeuteten einen starken Rückgang von Tätigkeiten wie Luftfahrt, Tourismus, Handel und Freihandelszonen, was zu Entlassungen, Arbeitsplatzverlusten und bestenfalls zu Lohn- und Gehaltkürzungen führte. Laut CEPAL (2020) sind fast 99 Prozent der Unternehmen in Lateinamerika Kleinst-, Klein- oder Mittelunternehmen

4 Informelle Beschäftigung ist eine Beschäftigung, die nicht unter das Arbeitsrecht fällt. 
(KKMU). Die pandemiebedingten Schließungen haben viele von ihnen nicht überlebt.

Ungleichheit und Prekarität kommen darüber hinaus vor allem in den Bereichen Gesundheit, Wohnen und Bildung zum Ausdruck. Schon vor der Pandemie waren die Bildungsunterschiede zwischen Kindern aus armen Familien und diesen aus wohlhabenden Familien groß. Das öffentliche Bildungswesen steckte in der Krise (Peters 2014). Die öffentlichen Gesundheitsausgaben liegen in der Region bei durchschnittlich 2,2 Prozent des BIP und damit bei der Hälfte dessen, was die WHO empfiehlt. Es besteht eine große Ungleichheit in Bezug auf den Zugang zu Gesundheitsversorgung. Angesichts endemischer Krankheiten wie Dengue, Malaria, Chagas oder dem jüngsten Wiederauftreten von Tuberkulose fallen die Ungleichheiten bezüglich der Bedingungen für ein gesundes Leben nochmals gravierender aus. Die Gesundheitssysteme waren schon immer chronisch unterfinanziert, fragmentiert, stratifiziert und segmentiert. Dies trifft auch auf die Anbindung an grundlegende infrastrukturelle Leistungen zu: 21 Prozent der Stadtbevölkerung hat keinen Zugang zu Wasser und 15 Millionen Menschen in der Region verrichten ihre Notdurft im Freien. In städtischen Gebieten lebt jeder vierte Mensch in informellen oder prekären Siedlungen. Das sind schätzungsweise 110 Millionen Menschen.

Die Feminisierung der Armut ist ein Nährboden für die Reproduktion von Ungleichheit. In Lateinamerika ist die Fertilitätsrate bei Jugendlichen fünfmal höher als in europäischen Ländern und der Anteil der Alleinerziehenden (17 Prozent) ist ebenfalls hoch. Die Zahl der Haushalte, die von alleinstehenden Frauen geführt werden, nimmt stetig zu (ECLAC/UNICEF 2014). Dies trifft vor allem auf die ärmsten Bevölkerungsschichten zu. Hier werden 30 Prozent der Haushalte von alleinstehenden Frauen geführt. Es sind diese Frauen, die für das Überleben sorgen, indem sie den Mangel an Dienstleistungen in den Armutsvierteln bekämpfen, Wassertanks und Nahrungsmittelhilfen beschaffen und Suppenküchen organisieren. Zudem sind Frauen zentral für die Aufrechterhaltung der Basisversorgung der lateinamerikanischen Gesellschaften. Sie stellen 80 Prozent der Beschäftigten im Verkauf und der Verarbeitung von Lebensmitteln und 75 Prozent der Beschäftigten im Gesundheitssektor (vgl. Kim in diesem Band).

Die Konzentration des Einkommens ist das Herzstück der lateinamerikanischen Ungleichheit. Die reichsten 10 Prozent der Bevölkerung verdienen 22-mal mehr als die ärmsten 10 Prozent besitzen, sie besitzen 71 Prozent des Reichtums und besteuern nur 5 Prozent ihres Einkommens (CEPAL 2020). Nach Berechnungen von Oxfam (2019) würden bei einer Fortsetzung dieses Trends die reichsten 1 Prozent der Region in nur sechs Jahren mehr Vermögen besitzen als die übrigen 99 Prozent. Zwischen 
2002 und 2015 wuchs das Vermögen der lateinamerikanischen Milliardär*innen um durchschnittlich 21 Prozent pro Jahr (siehe auch: Kaltmeier 2019).

Die lateinamerikanischen Staaten werden ihrer Umverteilungsfunktion offensichtlich nicht gerecht. Die Kultur der Privilegien naturalisiert Ungleichheiten und Diskriminierung und bekämpft soziale Probleme mit einem assistenzialistischen Wohlfahrtsstaat. Der lateinamerikanische Kapitalismus wurde als Hierarchie- oder Privilegienkapitalismus beschrieben, der auf der Vereinnahmung des Staates und seiner Vorschriften durch Eliten beruht, so dass Reichtum nicht nur wirtschaftliche Sicherheit, sondern auch soziale und politische Macht verleiht. Wirtschaftliche Ungleichheit verschärft die politische Ungleichheit und führt zu einem Teufelskreis, in dem die wohlhabenden Eliten ihre Ressourcen nutzen, um politische und wirtschaftliche Entscheidungen zu kontrollieren. Reichtum erkauft Einfluss und ermöglicht es den Eliten, sich über Generationen hinweg zu reproduzieren (Donald/Martens 2018).

\section{Regulierung, Umverteilung und Ungleichheit}

Die Gestaltung der Steuerpolitik ist für die Verteilung des Reichtums von entscheidender Bedeutung. Steuerpolitik kann nicht nur mehr Gleichheit erzeugen, sondern auch verhindern, dass Ungleichheit entsteht. Die Besteuerung sollte deshalb nicht nur als ein Instrument zur Generierung von finanziellen Mitteln für die Staaten zur Erfüllung ihrer Verpflichtungen verstanden werden, sondern auch als ein wirtschaftspolitisches Instrument zur gerechten Umverteilung des gesellschaftlichen Reichtums. Gerade dies findet in Lateinamerika jedoch nicht statt. Die Staatshaushalte hängen in erster Linie von der Ausbeutung natürlicher Ressourcen ab, deren Preise unbeständig sind und die große sozial-ökologische Probleme beinhalten. Die Einnahmen aus nicht erneuerbaren natürlichen Ressourcen machen einen beträchtlichen Teil der Gesamteinnahmen des öffentlichen Sektors aus; so betragen die Einnahmen aus Kohlenwasserstoffen beispielsweise 30 Prozent in Bolivien, 34 Prozent in Mexiko, 40 Prozent in Ecuador und 44 Prozent in Venezuela.

Die Steuern in Lateinamerika sind regressiv, d.h. sie belasten diejenigen stärker, die weniger haben, weil das Steuersystem hauptsächlich auf Verbrauchssteuern beruht, vor allem auf der Mehrwertsteuer. Insgesamt setzt sich die Standardsteuererhebung in Lateinamerika zu 46,2 Prozent aus Verbrauchssteuer, 26,8 Prozent aus Einkommenssteuer und 20,5 Prozent aus Beiträgen zur Sozialversicherung zusammen (ECLAC-OXFAM 2016). 
Verbrauchssteuern sind für die unteren und mittleren Einkommensgruppen, die keine Sparmöglichkeiten haben und ihr gesamtes Einkommen für den Verbrauch aufwenden besonders belastend.

Nach Angaben von OXFAM $(2014 ; 2020 a)$ werden Vermögen in Lateinamerika praktisch nicht besteuert und zudem ist Steuerhinterziehung weit verbreitet. Nur drei Länder in der Region haben irgendeine Form von Vermögenssteuer (Argentinien, Kolumbien und Uruguay). Im Ergebnis sind die Steuersysteme in der Region eher auf Arbeitseinkommen als auf Kapitalerträge ausgerichtet. Während Einkommenssteuern in letzter Zeit an Bedeutung gewonnen haben, werden Kapitalerträge und Erbschaften kaum besteuert. Allerdings ist die Einkommensteuer in Lateinamerika vor allem bei den höheren Einkommensgruppen niedrig. Dies liegt auch daran, dass die lateinamerikanischen Länder mehr als 50 Prozent ihrer Einnahmen aus den Einkommenssteuern durch Steuervermeidung und Steuerhinterziehung verlieren, schätzungsweise 38 Prozent in Mexiko, 49 Prozent in Argentinien und 58 Prozent in Ecuador. Zwischen 2002 und 2015 wuchs das Vermögen der lateinamerikanischen Milliardär*innen um durchschnittlich 21 Prozent pro Jahr. Im Jahr 2021 stehen 107 lateinamerikanische Milliardär*innen auf der Forbes-Liste, von denen die große Mehrheit ihr Vermögen im letzten Jahr, mitten in der Pandemie, vermehrt hat. Ein großer Teil dieses Vermögens wird steuerfrei oder in Steuerparadiesen gehalten.

Die Steuerbelastung der nationalen Unternehmen ist doppelt so hoch wie die der multinationalen Unternehmen, die von Steuerbefreiungen profitieren, um Investitionen anzulocken, die auch ohne diesen Anreiz kämen. Es gibt zudem eine Vielzahl von Möglichkeiten der Steuervermeidung. Die Steuerhinterziehung von Unternehmen reicht von 26 Prozent in Brasilien bis 65 Prozent in Ecuador. Steueroasen und der Missbrauch durch Unternehmen führen dazu, dass diejenigen, die einen größeren Beitrag zur Gesellschaft leisten könnten, nicht ihren Anteil leisten (CEPAL/ OXFAM 2016).

In den meisten Ländern der Region wird die schrittweise Anhebung des Steuersatzes und der mehrwertsteuerpflichtigen Waren fortgesetzt, anstatt die Steuerhinterziehung zu bekämpfen und die Steuerbefreiungen für diejenigen zu verringern, die am meisten besitzen. Zur Verringerung der Ungleichheiten sind umfassende Reformen der Steuersysteme erforderlich, um die direkten und indirekten Steuern wieder ins Gleichgewicht zu bringen, indem die Steuerlast von der Arbeit und dem Konsum auf Kapital, Vermögen und damit verbundene Einkommen zu verlagern. Dies kann beispielsweise durch Steuern auf Finanztransaktionen, Vermögen, Erbschaften und Kapitalerträge erfolgen. 


\section{Pandemiezeiten verschärfen die Ungleichheit}

Die Einschränkungen und Restriktionen zur Eindämmung der Pandemie haben die historischen Strukturprobleme Lateinamerikas verschärft: Ungleichheit, Informalität, Fragmentierung der Gesundheits- und Sozialschutzsysteme und begrenzter steuerlicher Spielraum haben in der Pandemie jeweils zugenommen.

Der Rückgang des BIP in der Region ist mit 7,7 Prozent der größte seit 120 Jahren. 52 Millionen Menschen sind unter die Armutslinie gerutscht. Die Armutsrate stieg von 30,3 Prozent im Jahr 2019 auf 37,3 Prozent im Jahr 2020 und die extreme Armut von 11,2 Prozent (2019) auf 15,6 Prozent (2020). Die wirtschaftliche Lage von rund 115 Millionen Menschen hat sich verschlechtert, 59 Millionen von ihnen gehörten 2019 der mittleren Einkommensschicht an. Der Gini-Index ist im Durchschnitt um 5,6 Prozent höher als im Jahr 2019 (CEPAL 2021).

Zu Beginn der Pandemie - ab Mitte März 2020 - haben fast alle Länder der Region Maßnahmen ergriffen, um die Bewegungsfreiheit einzuschränken und öffentliche Plätze zu schließen, den Unterricht auszusetzen, sowie die Wirtschaftstätigkeit einzuschränken. Zudem reagierten viele Länder mit Geldtransfers und Nahrungsmittelhilfen sowie teilweise auch mit Preiskontrollen und dem Verbot von Entlassungen, um dem Anstieg der Armut irgendwie zu begegnen. Dennoch ist die Abwärtsmobilität eindeutig: acht von zehn Lateinamerikaner*innen befinden sich in einer prekären sozialen Lage. Es ist daher notwendig, universelle Sozialprogramme voranzubringen. Denn der Anstieg der Armut infolge der COVID-19-Pandemie ist nicht auf das Jahr 2020 beschränkt, sondern wird auch in den Folgejahre etwa in Form höherer Informalität und Arbeitslosigkeit fortwirken.

Das gesellschaftliche Klima ist einerseits geprägt von Protesten und Unruhen. Andererseits zeigt sich ein starkes Gefühl sozialer Schutzlosigkeit und ein hohes Misstrauen gegenüber staatlichem Handeln, auch als Folge des bekannten Versagens des Staates. Der Umgang mit der Pandemie wird negativ wahrgenommen, sowohl mit Blick auf die gesundheits- als auch auf die sozialpolitischen Antworten. Die seit langem bestehende Frustration eines großen Teils der Bevölkerung kommt seit 2019 zum Ausdruck und auch in der Pandemie wehrt sich die Bevölkerung trotz der Umstände und fordert umfassende und klare Reformen, um Ungleichheiten und Armut zu verringern (vgl. Rojas in diesem Band). In der Zwischenzeit wurden in der Pandemie gemeinschaftliche, soziale und nichtstaatliche Solidaritätsnetze gestärkt.

Es kommt häufig zu kollektiven Mobilisierungen in den sozialen Netzwerken und im Internet sowie auf der Straße und zu sozialen Protesten 
gegen die soziale Krise. Besonders lautstark werden Forderungen für bessere Einkommensverteilung, soziale Absicherung, Pflege und universelle öffentliche Dienstleistungen artikuliert. Vor allem aber stehen Forderungen nach einem Grundeinkommen, Nothilfen und anderen Mechanismen, die den Lebensunterhalt ermöglichen, im Vordergrund der Forderungen.

Andererseits haben die Pandemie und der Lockdown zu Veränderungen in der Organisation des täglichen Lebens, der sozialen Beziehungen und anderen psychosozialen Aspekten geführt. Eine pandemische Müdigkeit macht sich breit: Zukunftsangst, Unbehagen angesichts der Ungewissheit, fehlende soziale Kontakte, Reizbarkeit und Müdigkeit. Derweil treiben die religiösen Fundamentalismen, die sich in unseren Gesellschaften in der Glut der Angst entwickelt haben, den Trend zum konservativen Backlash in Lateinamerika in verschiedenen Bereichen des gesellschaftlichen Lebens voran.

Die Pandemie hat eine neue Gruppe von verarmten Menschen und eine neue Form der akuten Ungleichheit zwischen denjenigen hervorgebracht, die weiterhin ihr Einkommen aus Arbeit beziehen, und denjenigen, die aufgrund gesundheitlicher Einschränkungen nicht arbeiten können oder ihren Arbeitsplatz verloren haben. Die Benachteiligung ist besonders grausam für diejenigen, die schon vorher arm waren. Internationale Organisationen haben vor dieser extremen Form von Ungleichheit und Armut und ihren explosiven Folgen gewarnt.

Sogar der Internationale Währungsfonds (IWF) zeigte sich besorgt, da er der Ansicht ist, dass die Wähler"innen bei zunehmender Ungleichheit dazu neigen, politische Entscheidungen zu treffen, die zu einer wachsenden Polarisierung bis hin zur Destabilisierung von Demokratien und Volkswirtschaften führen können. Zudem wird argumentiert, dass die Zunahme der Ungleichheit auf einen Rückgang der sozialen Mobilität zurückzuführen ist und dies Produktivität reduziere, weil der Anreiz zur Arbeitsanstrengung sinke (Barrett/Chen 2021).

Die Interamerikanische Entwicklungsbank (IDB) hat im Jahr 2020 davor gewarnt, dass die Krise arme und vulnerable Bevölkerungsgruppen unverhältnismäßig stark treffen wird. Vor diesem Hintergrund argumentiert die IDB, dass die Region ihre Umverteilungspolitik überdenken und sich auf die ärmsten Bevölkerungsschichten und insbesondere auf die öffentlichen Dienstleistungen konzentrieren müsse. Sie wies darauf hin, dass sich die Finanzpolitik in dieser Phase auf zwei Hauptziele konzentrieren sollte: (a) Bereitstellung ausreichender Mittel für den Gesundheitssektor, damit die gesundheitliche Notlage bewältigt werden kann, und (b) „Entlastungen“ für die am stärksten gefährdeten Haushalte sowie Maßnahmen zum Schutz der Unternehmen, die sie beschäftigen, um Massenentlassungen zu 
vermeiden. Es wird auch darauf hingewiesen, dass bei der Konzipierung dieser Maßnahmen zur Unterstützung von Privathaushalten und Unternehmen darauf geachtet werden muss, dass die eingeführten Transfers zeitlich begrenzt sind, da sie häufig nur schwer rückgängig gemacht werden können (Busso/Messina 2020).

Die CEPAL (2020) hält an der Notwendigkeit fest, ein Grundeinkommen für die am stärksten von der Pandemie betroffenen Sektoren beizubehalten. Zu diesem Zweck betont sie, dass es in einem Kontext wachsender Ungleichheit, niedriger Steuereinnahmen und regressiver Besteuerung notwendig ist, den Anwendungsbereich von Vermögens- und Substanzsteuern neu zu bewerten, die erneut im Mittelpunkt der globalen Debatte stehen.

Die Einkommensverteilung ist häufig das Ergebnis der Kräfteverhältnisse zwischen der einfachen Bevölkerung und der Elite, bei denen Geschichte, Politik und Institutionen ebenso eine Rolle spielen wie die volkswirtschaftlichen Grundlagen. Im Kontext der Covid-19 Pandemie manifestieren sich die Konflikte darüber, wie Reformen mit Blick auf Umverteilung, einer Umstrukturierung in Richtung solidarischerer Systeme und einer Besteuerung zur gerechteren Regulierung des Reichtums aussehen könnte. José Palma (2020) argumentiert, dass in Lateinamerika „bisher das ,eherne Gesetz der Oligarchien' wirkte, demzufolge entwicklungshemmende Institutionen dazu neigen, sich zu reproduzieren“, und, dass die Eliten kaum dazu bereit sein werden, Räume für Verteilungspolitiken zu öffnen, die sie nicht begünstigen. In der Tat haben sie präventive Maßnahmen ergriffen, die als Ausdruck der Rebellion aufgefasst wurden und den staatlichen Maßnahmen zur Ausweitung der Steuer- und Umverteilungsspielräume erwartungsgemäß Steine in den Weg legten. Benedicte Bull und Francisco Robles Rivera (2020) sind hingegen der Ansicht, dass, „wenn die Pandemiekrise die Eliten dazu zwinge, den Forderungen der benachteiligten Gruppen mehr Aufmerksamkeit zu schenken, dies möglicherweise zu einer Umstrukturierung der Institutionen [...] und langfristig zu einer Verringerung der Ungleichheit führen könnte“, was ein rationaleres Verhalten der Eliten sein könnte und der Verteilungsgerechtigkeit mehr Raum geben würde.

Doch bisher sieht das Bild anders aus. Auf der jährlichen Forbes-Liste ${ }^{5}$ der reichsten Menschen der Welt für das Jahr 2020 (veröffentlicht am 18. März 2020, genau als die Pandemie begann) geht hervor, dass es in Lateinamerika und der Karibik insgesamt 76 Milliardär*innen gibt, die zu-

5 https://www.forbes.com/billionaires/, letzter Aufruf 17.11.2020. 
sammen ein Nettogesamtvermögen von 284 Milliarden US-Dollar haben. Die Daten der Jahresliste 2021 (veröffentlicht am 5. März 2021, also ein Jahr nach der Pandemie) zeigen, dass diese Zahlen auf insgesamt 105 Milliardär*innen mit einem Gesamtnettovermögen von 448 Milliarden US-Dollar angestiegen sind. Schließlich verdeutlichen die jüngsten Daten der Echtzeitliste (bezogen auf den 17. Mai 2021), dass es einen erneuten Anstieg auf insgesamt 107 Milliardär*innen mit einem Gesamtnettovermögen von 480 Milliarden US-Dollar gab. Während der Pandemie ist die Zahl der Milliardär*innen in Lateinamerika und der Karibik mithin um 31 Prozent gestiegen und ihr gemeinsames Nettovermögen hat um 196 Milliarden US-Dollar zugenommen, was in etwa der Größe der ecuadorianischen Wirtschaft entspricht. Mit anderen Worten: Das Vermögen der lateinamerikanischen Milliardär*innen ist während der Covid-19 Pandemie um mehr als 40 Prozent gestiegen (López-Calva 2021).

\section{Sozioökonomische Maßnahmen zur Bekämpfung der Pandemiefolgen in Argentinien, Brasilien, Ecuador und Mexiko}

Im Folgenden werden die Ergebnisse einer Studie vorgestellt, die die sozioökonomischen Maßnahmen zur Bekämpfung der Pandemiefolgen in vier lateinamerikanischen Ländern (Argentinien, Brasilien, Ecuador und Mexiko) untersucht. Die Fallbeispiele wurden auf Basis der Bevölkerungszahl und der politischen Vielfalt ihrer Regierungen ausgewählt:

In Argentinien und Mexiko regierten zu Beginn der Pandemie progressive linksgerichtete Regierungen, die sich stärker auf die Bedürfnisse der ärmsten Bevölkerungsschichten konzentrierten, während Brasilien und Ecuador von neoliberalen, rechtsgerichteten Sektoren regiert wurden, deren Vorstellungen eher auf soziale Einschränkung und die Anhäufung von Reichtum mit geringer Umverteilung ausgerichtet waren. Die staatlichen Reaktionen auf die Pandemie reichten von der Verleugnung der Gefahren in Brasilien (siehe Eser, Vestena, Zhouri und Zilla in diesem Band), über strikte wirtschaftliche und soziale Maßnahmen in Argentinien bis hin zu teilweisen Schließungen in Mexiko und Ecuador.

Von den Vergleichsländern hat Brasilien den höchsten Einkommensanteil der reichsten 10 Prozent $^{6}$ und auch die ungleichste Einkommensvertei-

6 In keinem anderen demokratischen Land ist die Anhäufung von Einkommen bei den reichsten 1 Prozent größer. Als Ursachen gelten Privilegien, Patrimonialismus und Sklaverei. Folha de Sao Paulo vom 19. August 2019, https://temas.folha.uol.co 
lung, gefolgt von Mexiko. Laut World Inequity Data (2019) ist Brasilien das demokratischste Land mit der höchsten Einkommenskonzentration in den obersten 1 Prozent der Pyramide, mit 59,3 Prozent des Vermögens in den Händen der reichsten 10 Prozent und 31 Prozent in den Händen des reichsten 1 Prozent. In Mexiko befinden sich 58,6 Prozent des Vermögens in den Händen der reichsten 10 Prozent und 28,7 Prozent in den Händen der reichsten 1 Prozent. In Argentinien befinden sich 41,9 Prozent in den Händen der reichsten 10 Prozent und 17,8 Prozent in den Händen der reichsten 1 Prozent. In Ecuador befinden sich 37,6 Prozent in den Händen der reichsten 10 Prozent und 11,7 Prozent in den Händen der 1 Prozent. Darüber hinaus stieg das Nettovermögen der Milliardär*innen in der Frühphase der Pandemie laut OXFAM (2020b) zwischen dem 18. März und dem 12. Juli: in Brasilien um 30 Prozent und in Argentinien um 37 Prozent.

In allen Vergleichsländern ist der Anteil der informell Beschäftigten hoch, wobei Ecuador das Land ist, in dem die meisten der Arbeitskräfte im informellen Sektor tätig sind. Hinzu kommt ein Teil der Arbeitnehmer*innen, die zwar im formellen Sektor tätig sind, aber auch keinen Krankenund Rentenversicherungsschutz haben.

Laut dem World Values Survey (Haerpfer et al. 2020) gaben 52,2 Prozent der Lateinamerikaner*innen an, dass ihr Lebensstandard gleich oder schlechter sei als der ihrer Eltern. Dies deutet auf eine pessimistische Gegenwartswahrnehmung hin, während gleichzeitig die Wahrnehmung der Ungerechtigkeit bei der Einkommensverteilung zugenommen hat. Für die untersuchten Länder ergaben sich folgende Ergebnisse:

\section{Tabelle 1: Bewertung der Einkommensverteilung}

\begin{tabular}{|l|c|c|}
\hline & \multicolumn{2}{|c|}{$\begin{array}{l}\text { Personen, die die Einkommensverteilung für ungerecht er- } \\
\text { klären (Angaben in Prozent) }\end{array}$} \\
\hline & $\mathbf{2 0 1 3}$ & $\mathbf{2 0 1 9}$ \\
\hline Ecuador & 39 & 70 \\
\hline Brasilien & 81 & 92 \\
\hline Mexiko & 80 & 88 \\
\hline Argentinien & 75 & 90 \\
\hline
\end{tabular}

Quelle: Eigene Darstellung auf Basis von PNUD (2019) und Haerpfer et al. (2020).

m.br/desigualdad-global/brasil/los-super-ricos-en-brasil-lideran-la-concentracion-dela-renta-global.shtml, letzter Aufruf 30.12.2021. 
Die Wahrnehmung von Ungerechtigkeit hat ab 2019 stark zugenommen und ist in Zeiten der Pandemie sicherlich noch stärker geworden. Gleichzeitig stieg die Armut während der Pandemie (Tabelle 2).

Tabelle 2: Entwicklung der Armut von 2019 bis 2020 (Angaben in Prozent)

\begin{tabular}{|c|c|c|c|}
\hline & Armut 2019 & Armut 2020 & Veränderung \\
\hline Ecuador & 25,7 & 33,6 & $+7,9$ \\
\hline Brasilien & 19,3 & 24,1 & $+4,8$ \\
\hline Mexiko & 41,5 & 50,6 & $+9,1$ \\
\hline Argentinien & 27,2 & 38,8 & $+11,6$ \\
\hline
\end{tabular}

Quelle: Eigene Darstellung auf der Grundlage von CEPAL-Daten.

In allen vier Ländern wurden als Reaktion auf die Pandemie wirtschaftliche Maßnahmen ergriffen, die sich an Unternehmen und arme bzw. vulnerable Bevölkerungsgruppen richteten (Tabelle 3).

\section{Tabelle 3: Maßnabmen zur Abfederung der Auswirkungen der Corona- Pandemie}

\begin{tabular}{|l|l|}
\hline MEXIKO & ECUADOR \\
\hline $\begin{array}{l}\text { Die Sozialversicherungsanstalt verschiebt die } \\
\text { Zahlungen an die Sozialversicherung für April, } \\
\text { Mai und Juni um 90 Tage. }\end{array}$ & Aussetzung von Steuerverwaltungsverfahren \\
\hline $\begin{array}{l}\text { Senkung des Zielwerts für den Tagesgeldsatz } \\
\text { um 50 Basispunkte auf ein Niveau von 6,5 Pro- } \\
\text { zent (von 7,0 Prozent im Februar 2020) }\end{array}$ & $\begin{array}{l}\text { Die Sozialversicherungsanstalt verschiebt die } \\
\text { Zahlungen an die Sozialversicherung für April, } \\
\text { Mai und Juni um 90 Tage. }\end{array}$ \\
\hline $\begin{array}{l}\text { Stärkung der Kreditvergabekanäle und Bereit- } \\
\text { stellung von Liquidität für eine gesunde Ent- } \\
\text { wicklung des Finanzsystems }\end{array}$ & $\begin{array}{l}\text { Gesetz zur Ordnung der öffentlichen Finanzen } \\
\text { Auflösung der staatlichen Unternehmen: Eisen- } \\
\text { bahn, Plantagen, öffentliche Medien, Crear, } \\
\text { Ecuador Estratégico, Correos de Ecuador (Post), } \\
\text { Unidad de Almacenamiento und TAME. Staat- } \\
\text { liche Unternehmen, die nicht aufgelöst werden, } \\
\text { werden ihre Ausgaben erheblich reduzieren. }\end{array}$ \\
\hline $\begin{array}{l}\text { Investitionen in öffentliche Bau- und Infra- } \\
\text { strukturprojekte }\end{array}$ & $\begin{array}{l}\text { Erhöhung des Preises für Extra- und Ecopais- } \\
\text { Benzin von 1 USD pro Liter auf 1,75 USD pro } \\
\text { Liter }\end{array}$ \\
\hline & $\begin{array}{l}\text { Verlängerung von Krediten und Stundung von } \\
\text { Quoten }\end{array}$ \\
\hline & Genehmigung von Kurz- oder Teilzeitarbeit \\
\hline & $\begin{array}{l}\text { Humanitäres Gesetz, das die Beschäftigungsbe- } \\
\text { dingungen, die Löhne und die Verhandlung } \\
\text { von Konflikten wesentlich flexibler gestaltet }\end{array}$ \\
\hline
\end{tabular}




\begin{tabular}{|c|c|}
\hline ARGENTINIEN & BRASILIEN \\
\hline $\begin{array}{l}\text { Unterstützung für Arbeit und Produktion (Asis- } \\
\text { tencia al Trabajo y la Producción (ATP) } \\
\text { 2,3 Millionen Erwerbstätige erhielten Unter- } \\
\text { stützungen für die Gehälter }\end{array}$ & $\begin{array}{l}\text { Ausweitung der Kreditvergabe durch das Fi- } \\
\text { nanzsystem, wodurch die Kreditvergabe an } \\
\text { Kleinst- und Kleinunternehmen im Vergleich } \\
\text { zu } 2019 \text { um mehr als } 37 \text { Prozent gesteigert wer- } \\
\text { den konnte, was 1,7 Prozent des BIP entspricht. }\end{array}$ \\
\hline $\begin{array}{l}\text { Programm zur produktiven Erholung 2: Dieses } \\
\text { Programm besteht darin, dass den Arbeitneh- } \\
\text { mer*innen ein individueller und fester Betrag } \\
\text { gezahlt wird, der zu Lasten der Arbeitgeber*in- } \\
\text { nen geht }\end{array}$ & $\begin{array}{l}\text { Zahlungsaufschub der Sozialversicherungsbei- } \\
\text { träge und der Arbeitgeberbeiträge zur Sozial- } \\
\text { versicherung }\end{array}$ \\
\hline $\begin{array}{l}\text { Vorübergehende Befreiung von den Sozialversi- } \\
\text { cherungsabgaben für die am stärksten betroffe- } \\
\text { nen Wirtschaftssektoren }\end{array}$ & Senkung des Steuersatzes auf Kredite \\
\hline $\begin{array}{l}\text { Erhöhung der öffentlichen Infrastrukturinvesti- } \\
\text { tionen }\end{array}$ & $\begin{array}{l}\text { Die Steuern auf eingeführte Vorleistungen, die } \\
\text { zur Herstellung der Ausfuhrware verwendet } \\
\text { werden, werden ausgesetzt oder aufgehoben. }\end{array}$ \\
\hline $\begin{array}{l}\text { Erweiterung einer Kreditlinie zur Förderung } \\
\text { des Wohnungsbausektors }\end{array}$ & $\begin{array}{l}\text { Senkung der Mindestreserveanforderungen der } \\
\text { Banken }\end{array}$ \\
\hline $\begin{array}{l}\text { Neuer Zahlungsaufschub für die Ausfuhrabga- } \\
\text { ben für KKMU }\end{array}$ & $\begin{array}{l}\text { PROGER/FAT-Kredit für Kleinst- und Kleinun- } \\
\text { ternehmen }\end{array}$ \\
\hline $\begin{array}{l}\text { Neue Sonderkreditlinie für KMU und Erweite- } \\
\text { rung des bestehenden Programms für zinsgüns- } \\
\text { tige Darlehen für den privaten Verbrauch (Pro- } \\
\text { gramm „Ahora 12“), }\end{array}$ & $\begin{array}{l}\text { Stundung der Zahlungen im "Simples Nacio- } \\
\text { nal“" }\end{array}$ \\
\hline
\end{tabular}

Quelle: Eigene Darstellung auf Grundlage der Covid-Beobachtungsstelle der CEPAL, https://www.cepal.org/es/temas/covid-19, letzter Aufruf 30.12.2021

In allen vier Ländern wurden Steuerbefreiungen eingeführt und verspätete Sozialversicherungszahlungen, Steueraussetzungen und Beitragsstundungen ermöglicht. Zudem wurde die Kreditvergabe ausgeweitet und in Argentinien Kredite für KKMU erleichtert. In Ecuador wurden unter dem kuriosen Titel „Humanitäres Gesetz“ (Ley Humanitaria) die Regularien für die Einstellung von Arbeitskräften gelockert, was den Unternehmen zugutekam und den Arbeitnehmer*innen schadete, da es die Instabilität ihrer Arbeitsplätze fördert. Hinzu kommt der Widerstand gegen die Erhöhung der Kraftstoffpreise, die sowohl kleine Unternehmen als auch die ärmsten Sektoren trifft.

In der folgenden Tabelle sind die Ausgleichsmaßnahmen für Personen aufgeführt, die am stärksten von prekären Beschäftigungsverhältnissen und Armut betroffen sind: 


\section{Tabelle 4: Ausgleichsmaßnahmen für besonders vulnerable Personen}

\begin{tabular}{|c|c|}
\hline MEXIKO & ECUADOR \\
\hline Mikrokredite für kleine Familienunternehmen & $\begin{array}{l}\text { Bono de Protección Familiar por Emergencia } \\
\text { Einmalige Transferleistung - gezahlt in zwei } \\
\text { Raten - von } 120 \text { USD, für Personen mit einem } \\
\text { Einkommen unterhalb des Mindestlohns, un- } \\
\text { bezahlte Hausangestellte und an Mitglieder des } \\
\text { Versicherungssystems für Bäuer*innen richtet. } \\
\text { Die Transferleistungen wurden in den Jahren } \\
2020 \text { und } 2021 \text { ausgezahlt. }\end{array}$ \\
\hline $\begin{array}{l}\text { Vorgezogene Altersrenten für ältere Menschen } \\
\text { und Menschen mit Behinderungen }\end{array}$ & $\begin{array}{l}\text { Wiedereingliederung von Familien, die keine } \\
\text { Sozialversicherungsbeiträge mehr zahlen }\end{array}$ \\
\hline Vorauszahlung von Stipendien für Studierende & $\begin{array}{l}\text { Arbeitslosenunterstützungsbonus in Höhe } \\
\text { von } 500 \text { US-Dollar }\end{array}$ \\
\hline $\begin{array}{l}\text { Wirtschaftliche Unterstützung für den länd- } \\
\text { lichen Raum: („Sembrando Vida en Coronavi- } \\
\text { rus“) }\end{array}$ & $\begin{array}{l}\text { Verbot der Aussetzung der Grundversorgung } \\
\text { und der Zwangsräumung bei Nichtzahlung }\end{array}$ \\
\hline ARGENTINIEN & BRASILIEN \\
\hline $\begin{array}{l}\text { Familiennotgeld (Ingreso Familiar de Emergen- } \\
\text { cia) (IFE), das 8,4 Millionen Menschen (infor- } \\
\text { mell Beschäftigte, Selbstständige mit geringem } \\
\text { Einkommen, Arbeitslose und Nichterwerbstäti- } \\
\text { ge) dreimal gewährt wird und nur von einem } \\
\text { Familienmitglied bezogen werden kann }\end{array}$ & $\begin{array}{l}\text { Soforthilfe der brasilianischen Bundesregie- } \\
\text { rung: Finanzielle Unterstützung in bis zu drei } \\
\text { Raten: } \\
\text { 1. } 600 \mathrm{R} \$ \text { Monat für bis zu zwei Personen der } \\
\text { gleichen Familie } \\
\text { 2. } 1.200 \mathrm{R} \$ \text { /Monat für weibliche Haushalts- } \\
\text { vorstände. } \\
60 \text { Millionen Menschen erhielten diese Mittel }\end{array}$ \\
\hline $\begin{array}{l}\text { Allgemeines Kindergeld (Asignación Universal } \\
\text { por Hijo) (AUH), dessen Umfang um 8,7 Pro- } \\
\text { zent zunahm }\end{array}$ & $\begin{array}{l}\text { Wiederaufnahme im Jahr } 2021 \text { mit } 15 \text { Prozent } \\
\text { geringeren Leistungen und } 22 \text { Millionen weni- } \\
\text { ger Leistungsempfänger"innen }\end{array}$ \\
\hline \multicolumn{2}{|l|}{ Schwangerschaftsgeld (AUE) } \\
\hline \multicolumn{2}{|l|}{$\begin{array}{l}\text { Die Kürzung der Grundversorgung wurde für } \\
\text { drei Monate untersagt }\end{array}$} \\
\hline \multicolumn{2}{|l|}{$\begin{array}{l}\text { Zahlungsaufschub für das Rentner*innendarle- } \\
\text { hen um zwei Monate }\end{array}$} \\
\hline $\begin{array}{l}\text { Verlängerung der Einkommenssteuerbefreiung } \\
\text { für Personal des Gesundheitswesens, des Si- } \\
\text { cherheitsdienstes und anderer systemrelevanter } \\
\text { Dienste }\end{array}$ & \\
\hline
\end{tabular}

Quelle: Eigene Darstellung auf Grundlage der COVID-Beobachtungsstelle der CEPAL, https://www.cepal.org/es/temas/covid-19, letzter Aufruf 30.12.2021

In allen vier Ländern wurde die Bevölkerung in irgendeiner Form unterstützt, wobei der Schwerpunkt auf den armen und vulnerablen Haushalten lag. Mexiko konzentrierte sich auf Stipendien- und Rentenvorschüsse, Unterstützungsleistungen für den ländlichen Raum sowie Mikrokredite. Das Land stellte keine Sondermitteln - wie Ecuador, Argentinien und Brasilien - ohne Rückerstattung für informell Beschäftigte bereit. Die von Ecuador bereitgestellten Mittel waren sowohl in Bezug auf die Höhe 
als auch den Umfang gering und hinsichtlich der Sicherheit für die betroffenen Bevölkerungsgruppen unzureichend. Argentinien und Brasilien konzentrierten sich auf die Unterstützung von informell Beschäftigten, Arbeitslosen, alleinstehenden Müttern mit minderjährigen Kindern und minderjährigen Müttern. In Brasilien wurden monatliche Hilfszahlungen eingeführt und in Argentinien wurden entsprechende Zahlungen in drei Raten getätigt. Brasiliens Nothilfen erreichten im Jahr 202060 Millionen Personen. Diese Zahl reduzierte sich allerdings im Jahr 2021. Diese Nothilfeprogramme in Brasilien und Argentinien waren wichtig für die Stabilisierung der Wirtschaft und für die Aufrechterhaltung des sozialen Friedens.

Die notwendigen finanziellen Anstrengungen, die die Länder zur Deckung der höheren Gesundheitsausgaben zur Bewältigung der Pandemie sowie für die Nothilfen unternehmen mussten, steigerte die Notwendigkeit höhere Steuereinnahmen zu generieren und progressivere Steuersysteme zu errichten. In diesem Sinne verabschiedete Argentinien am 4. Dezember 2020 das Gesetz über die Solidarität und den außerordentlichen Beitrag der großen Vermögen (Ley de aporte solidario y extraordinario de las grandes fortunas). Dieser Beitrag wurde als außerordentlicher, einmaliger Solidaritätsbeitrag definiert, der an das Vermögen von Privatpersonen und ungeteilten Nachlässen mit einem Vermögen von mehr als 200 Millionen argentinischen Pesos (2.100.000 US-Dollar) geknüpft ist. Der Steuersatz für im Land deklariertes Vermögen beträgt zwischen 2 Prozent und 3,5 Prozent $^{7}$. Das Gesetz selbst legt fest, wofür die Einnahmen verwendet werden sollen: 20 Prozent für den Kauf und/oder die Herstellung medizinischer Ausrüstung, Schutzausrüstungen, Arzneimitteln, Impfstoffe etc., 20 Prozent für Subventionen für KKMU, 20 Prozent für Stipendien für Studierende, 15 Prozent für den Fonds für sozio-urbane Integration (Fondo de Integración Socio Urbana) zur Verbesserung der Wohnbedingungen für die Bewohner*innen armer Stadtviertel und 25 Prozent für die Erkundung, Erschließung und Förderung von Erdgas. Diese letztgenannte Zuweisung wurde von den Gewerkschaften und sozialen Bewegungen kritisiert.

Etwa 10.000 Personen mit einem Vermögen von mehr als 200 Millionen Pesos zahlten die Steuer. 20 Prozent verweigerten die Zahlung der Steuer und einige von ihnen legten Rechtsmittel ein. Der Staat nahm auf diese Weise 2,38 Milliarden Dollar ein. In einer vorläufigen Bewertung

7 Die erhebliche Steuerhinterziehung durch Nichtangabe von Vermögenswerten im Land und die übliche Angewohnheit der argentinischen Eliten, Gelder aus dem Land zu schicken, sollten berücksichtigt werden. 
kann die Maßnahme als moderat bewertet werden, da es sich um eine einmalige Abgabe handelte. Zudem kann sie mit Blick auf den erhobenen Betrag als erfolgreich eingeschätzt werden. Allerdings versuchen die Eliten weiterhin die eine steuerliche Umstrukturierung und Umverteilung zu erschweren. Dennoch gilt es auf eine Leerstelle hinzuweisen. Es erscheint kaum möglich mit einmaligen Maßnahmen Mittel zur Finanzierung wiederkehrender Ausgaben aufzubringen.

\section{Umverteilungsmöglichkeiten während der Pandemie}

Die Reduzierung von Ungleichheiten ist kein Selbstläufer, sondern erfordert aktives Handeln. In Lateinamerika sind die Ungleichheiten und die ungerechte Verteilung nicht nur extrem in ihren materiellen Manifestationen, sondern auch ideologisch manifestiert. Das heißt, es gibt eine historische Akzeptanz der Ungleichheit und der sozialen Not.

Die Notwendigkeit eines neuen Sozialpakts, der das Recht auf Existenzsicherung und auf ein garantiertes Mindesteinkommen als grundlegendes Menschenrecht beinhaltet, ist in der Welt und erst recht in Lateinamerika noch lange nicht selbstverständlich. Trotz wiederholter Aufforderungen, die Soforthilfen fortzusetzen, sind wir angesichts der Bedingungen in der Region weit davon entfernt, die bereits bestehende und durch die Pandemie noch verschärfte Krise zu lindern.

Die sozialen Unruhen in der Region fordern ein Ende der Kultur der Privilegien. Insbesondere eine Steuerreform ist notwendig, um mehr Verteilungsgerechtigkeit zu erreichen. Doch der erbitterte Widerstand der großen Vermögen gegen außerordentliche Beiträge zur Linderung der verheerenden Pandemie spricht nicht nur für das Ausmaß der sozialen Ungerechtigkeit in unseren Gesellschaften, sondern auch für die Orientierungslosigkeit und Kurzsichtigkeit der sie beherrschenden Eliten. In diesem Kontext verschärfen sich bestehende Verteilungskonflikte und große Teile der Bevölkerung mobilisieren sich. Für die Zukunft ergeben sich Szenarien, die von Reformen bis zu gewalttätigen Konflikten reichen.

Die lateinamerikanischen Eliten teilen ein Gefühl, das auch die Mittelschichten durchdringt und sich in folgenden Punkten zusammenfassen lässt: ein Ideal des Minimalstaates und die Delegitimierung des Staates; die weit verbreitete Überzeugung, dass höhere Sozialabgaben die Situation nicht verbessern werden; Strategien zur Verhinderung einer gerechteren Besteuerung; sowie ein extraktiver Kapitalismus, der nicht auf der Inwertsetzung von Humankapital basiert. Angesichts dieser weit verbreiteten Überzeugungen sollten die Bereitstellung öffentlicher Güter und die 
staatliche Rechenschaftspflicht verbessert werden, um das Vertrauen zu stärken und so vielleicht eine Neustrukturierung des Steuersystems und damit mehr Verteilungsgerechtigkeit zu erreichen. Aber hier stellt sich ein zirkuläres Problem: Bedingungen, die die Überzeugungen bestimmen und Überzeugungen, die Bedingungen erzeugen.

Vor diesem Hintergrund stellt sich die Frage, in welchen Fällen die lateinamerikanischen Eliten tendenziell eher bereit wären, Umverteilungsreformen zu akzeptieren. Grundsätzlich könnte dies das Ergebnis von Verhandlung zwischen politischen und wirtschaftlichen Entscheidungsträger*innen sein. Außerdem könnte ein gewisses Verantwortungsbewusstsein auf die Ungleichheit übertragen werden, um den Zusammenhang zwischen Unsicherheit und Ungleichheit aufzuzeigen. Andererseits könnte es am Rande von sozialen Unruhen und damit verbundenen traumatischen Ereignissen zu einer Lockerung der von den oberen Schichten aufrechterhaltenen geschlossenen Interessenvertretung kommen.

Die Interamerikanische Entwicklungsbank hat darauf hingewiesen, dass es trotz der gesundheitlichen Notlagen, mit denen die Länder konfrontiert sind, wichtig ist, zu bedenken, dass es in Krisenzeiten mehr Möglichkeiten gibt, wirtschaftliche und soziale Maßnahmen zu ergreifen, die die Interessen der gesamten Gesellschaft in den Vordergrund stellen. Das Bewusstsein für die außergewöhnliche Dimension dieser Krise und die Lehren, die aus einem globalen Virus zu ziehen sind, könnte die Einsicht für die Notwendigkeit tiefgreifender Veränderungen schärfen. Ein Anfang könnte die jüngste G7-Vereinbarung von Anfang Juni 2021 über Steuerinternationalismus sein. Diese zielt darauf ab Steuerverlagerungen zu verhindern und mit einer globalen Mindeststeuer zur Finanzierung öffentlicher Güter beizutragen.

\section{Literatur:}

Alarco Tosoni, Germán; Castillo García, César (2020): Concentración de la riqueza en América Latina en el siglo XXI, in: Prob. Des, 51, 111-136.

Alba Vega, Carlos (2021): Las relaciones de los empresarios organizados con el presidente de México durante la pandemia, in: Desacatos, 65, 156-177.

Banco Mundial (2020): La pobreza y la prosperidad compartida 2020: Un cambio de suerte, Washington DC: Banco Mundial.

Barrett, Philip; Chen, Sophia (2021): Social Repercussions of Pandemics. IMF Working Paper, Washington DC: IMF [https:/www.imf.org/en/Publications/WP/Issues/2 021/01/29/Social-Repercussions-of-Pandemics-50041], letzter Aufruf 14.07.2021. 
Base de datos mundial sobre la desigualdad (2021): [https:/wid.world/data/], letzter Aufruf01.10.2021.

Bautista, Carolina (2020): Estados Alterados: reconfiguraciones estatales, luchas políticas y crisis orgánica en tiempos de pandemia, Buenos Aires: CLACSO.

BID; Pessino, Carola; Izquierdo, Alejandro (2021): Una estrategia fiscal post COVID-19 para América Latina y el Caribe, in: BID vom 07.04.2021 [https://blogs.iad b.org/ideas-que-cuentan/es/una-estrategia-fiscal-post-covid-19-para-america-latina -y-el-caribe/], letzter Aufruf 14.07.2021.

BID; Izquierdo, Alejandro et al. (2021): Salir del túnel pandémico con crecimiento y equidad: Una estrategia para un nuevo pacto social en América Latina y el Caribe, [https://publications.iadb.org/publications/spanish/document/Salir-del-tunel-pa ndemico-con-crecimiento-y-equidad-Una-estrategia-para-un-nuevo-compacto-soc ial-en-America-Latina-y-el-Caribe.pdf], letzter Aufruf 01.10.2021.

Bull, Benedicte; Robles Rivera, Francisco (2020): El COVID-19, las élites y el futuro de la economía política de la reducción de la desigualdad en América Latina, in: Revista de la CEPAL, 132, 79-94.

Burchardt, Hans-Jürgen; Groisman, Fernando (2014): Desprotegidos y desiguales. ¿Hacia una nueva fisonomía social?, Buenos Aires: Prometeo.

Busso, Matías; Messina, Julián (2020): La crisis de la desigualdad: América Latina y el Caribe en la encrucijada, [https://publications.iadb.org/publications/spanish/docu ment/La-crisis-de-la-desigualdad-America-Latina-y-el-Caribe-en-la-encrucijada.pd f], letzter Aufruf 01.10.2021

CEPAL (2016): Tributación para un crecimiento inclusivo, Santiago de Chile: CEPAL.

CEPAL (2020): Informe Especial COVID-19 No. 3. The social challenge in times of COVID-19, [https://repositorio.cepal.org/handle/11362/45527], letzter Aufruf 10.07.2021.

CEPAL (2021): Panorama Social 2020. Naciones Unidas, Santiago de Chile: CEPAL.

CEPAL (2021a): La protección social de los trabajadores informales ante los impactos del COVID-19, Santiago de Chile: CEPAL.

CEPAL; UNICEF (2014): La evolución de las estructuras familiares en América Latina, 1990-2010 Los retos de la pobreza, la vulnerabilidad y el cuidado, Naciones Unidas.

CEPAL; OXFAM (2016): Tributación para un crecimiento inclusivo, [https://www.oxf ammexico.org/sites/default/files/InformaES.pdf], letzter Aufruf 15.05.2021.

Haerpfer, Christian W., et al. (2020): World Values Survey: Round Seven, World Values Survey.

IMF (2021): Social Repercussions of Pandemics. IMF Working Papers.

Kaltmeier, Olaf (2019): Refeudalización. Desigualdad social, economía y cultura politica en América Latina en el temprano siglo XXI, Bielefeld: Bielefeld University Press - CALAS.

Latindadd (2020): Ahora o nunca: Impuestos a la riqueza y las grandes fortunas en América Latina y el Caribe, [http://impuestosalariqueza.org/wp-content/uploads/2 020/12/Informe-Impuestos-a-la-Riqueza.pdf], letzter Aufruf 01.10.2021. 
Lomelí Vanegas, Leonardo (2020): La economía de la salud en México, in: Revista de la CEPAL, 132, 195-208.

López Accotto, Alejandro; Martínez, Carlos R.; Mangas, Martín; Paparas, Ricardo (2019): Los impuestos a la riqueza en Argentina en una perspectiva comparada, in: Revista Econonomía y Desafíos del Desarrollo, I, http://www.unsam.edu.ar/escu elas/economia/revistaedd/3n $2 /$.

López-Calva, Luis Felipe (2021): COVID-19 y la riqueza en la cima: más multimillonarios y más ricos en ALC tras la crisis, [https:/www.latinamerica.undp.org/content/ rblac/es/home/presscenter/director-s-graph-for-thought/covid-19-and-wealth-at-th e-top--more-and-wealthier-billionaires-html], letzter Aufruf 01.10.2021.

OXFAM (2014): Justicia fiscal para reducir la desigualdad en Latinoamérica y el Caribe, [https://www-cdn.oxfam.org/s3fs-public/file_attachments/justicia_fiscal_para _reducir_la_desigualdad_en_latinoamerica_y_el_caribe_.pdf], letzter Aufruf 01.10.2021.

OXFAM (2020a): Vivir al día: medidas para combatir la epidemia de desigualdad en México, [https://www.oxfammexico.org/sites/default/files/Boleti\%CC\%81n\% 20de\%20prensa\%20-\%20Vivir\%20al\%20di\%CC\%81a_SO.pdf], letzter Aufruf 30.12.2021.

OXFAM (2020b): Nota de prensa: Aumentan los milmillonarios de América Latina a medida que la región más desigual del mundo se bunde bajo el impacto del coronavirus, [https://www.oxfam.org/es/notas-prensa/aumentan-los-mil-millonarios-de-a merica-latina-medida-que-la-region-mas-desigual], letzter Aufruf 30.07.2020.

OXFAM; CEPAL (2016): Tributación para un crecimiento inclusivo, Santiago de Chile: CEPAL.

Palma, José Gabriel (2020): Por qué los ricos siempre siguen siendo ricos (pase lo que pase, cueste lo que cueste), in: Revista de la CEPAL, 132, 95-138.

Peters, Stefan (2014): Educación, desigualdades y empleo: los límites de la expansión educative, in: Burchardt, Hans-Jürgen; Groisman, Fernando (Hg.): Desprotegidos y desiguales. ¿Hacia una nueva fisonomía social?, Buenos Aires: Prometeo, 185-207.

Piketty, Thomas (2014): El capital en el siglo XXI, Bogotá: Fondo de Cultura Económica.

PNUD (2019): Informe sobre Desarrollo Humano 2019. Más allá del ingreso, más allá de los promedios, más allá del presente: Desigualdades del desarrollo humano en el siglo XXI, [http://hdr.undp.org/sites/default/files/hdr_2019_overview_-_spanish.p df], letzter Aufruf 01.10.2021.

Schorr, Martín (2021): El viejo y el nuevo poder económico en la Argentina: del siglo XIX a nuestros días. Buenos Aires: Siglo XXI. 